\title{
Regulatory RNA-assisted Genome Engineering in Microorganisms
}

4

$15 *$ To whom correspondence should be addressed: 
Microbial genome engineering by trans-acting RNAs and accessory proteins

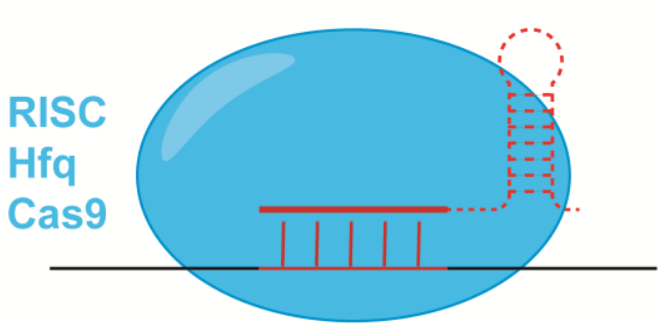

Target sequence: DNA or mRNA

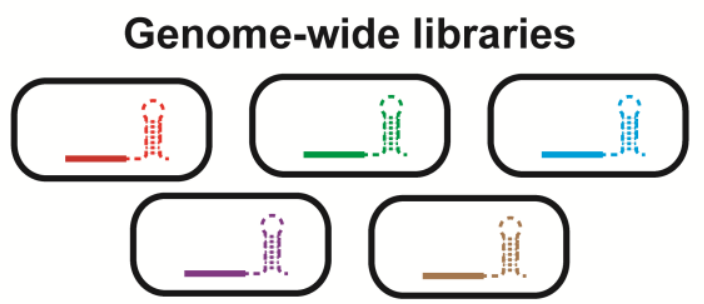

Combinatorial multiplex libraries
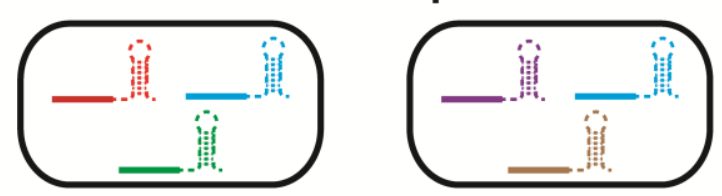

24

25 


\section{Abbreviations}

28 API: application program interface

29 asRNA: antisense RNA

30 CRISPR-Cas: clustered regularly interspaced short palindromic repeats and CRISPR associated proteins

31 CRISPRi: CRISPR interference

32 crRNA: CRISPR RNA

33 dCas9: deactivated Cas9

34 DR: direct repeat

35 DSB: double stranded break

36 HI-CRISPR: homology-integrated CRISPR-Cas

37 HR: homologous recombination

38 LACE: light-activated CRISPR-Cas9 effector

39 MAGE: multiplex automated genome engineering

40 NHEJ: non-hmologous end joining

41 PAM: protospacer adjacent motif

42 RAGE: RNAi-assisted genome engineering

43 RNAi: RNA interference

44 sRNA: small RNA

45 sgRNA: single guide RNA

46 TIR: translation initiation region

47 tracrRNA: trans-activating CRISPR RNA 
50 Regulatory RNAs are increasingly recognized and utilized as key modulators of gene expression in diverse 51 organisms. Thanks to their modular and programmable nature, trans-acting regulatory RNAs are especially 52 attractive in genome-scale applications. Here we discuss the recent examples in microbial genome 53 engineering implementing various trans-acting RNA platforms, including sRNA, RNAi, asRNA and 54 CRISRP-Cas. In particular, we focus on how the scalable and multiplex nature of trans-acting RNAs has 55 been used to tackle the challenges in creating genome-wide and combinatorial diversity for functional 56 genomics and metabolic engineering applications. Advances in computational design and context57 dependent regulation are also discussed for their contribution in improving fine-tuning capabilities of trans58 acting RNAs. 
61 Engineered microorganisms are emerging as solutions to many global challenges that we are facing today

62 by providing environmental remediation, medical applications, and renewable chemical production [1-3].

63 To achieve these engineering objectives, intensive reprogramming of cellular networks is needed, especially upon the introduction of foreign functionalities such as heterologous pathways and synthetic gene circuits. Given the complexity of biological systems, cellular reprogramming requires both identification of genetic targets on a genome scale and optimization of their expression in a concerted manner (multiplex editing). Such practices underlie the concept of "genome engineering", in which largescale and multiplex genetic modifications are created, in contrast with the more traditional term "genetic engineering", in which only a small number of genes are modulated [4-8].

Within this context, regulatory RNAs are increasingly implemented for comprehensive understanding and

71 engineering of genomes $[9,10 \bullet \bullet, 11]$. Interacting with DNA, RNA, protein and metabolite molecules, RNAs 72 can regulate every step of gene expression [11]. Specifically, trans-acting RNA-mediated gene regulation 73 is scalable and predictable, as the recognition of target DNA or RNA sequences is primarily governed by 74 the Watson-Crick base-pairing principle [6,10••]. Nowadays, genome-wide libraries of RNA molecules or 75 RNA expression cassettes can be readily designed and manufactured, facilitating screening and 76 identification of relevant genes to a given phenotype. Moreover, it is possible to introduce several 77 regulatory RNAs simultaneously in one cell and hence enable multiplex targeting. This capability is critical 78 in understanding and engineering non-linear interactions within complex genetic networks, as exemplified 79 as combinatorial optimization of multiple gene targets $[12,13]$.

80 In this review, we first survey examples of various trans-acting RNA regulatory mechanisms in microbial 81 genome engineering, including small RNA (sRNA), antisense RNA (asRNA), RNA interference (RNAi) 82 and the clustered regularly interspaced short palindromic repeats (CRISPR) and CRISPR associated 83 proteins (Cas) system. Both genome-scale screening and multiplex targeting applications will be 
emphasized. We then discuss some notable technological advances in implementing RNA-based genome engineering, including computational design and context-dependent regulation.

\section{Trans-acting regulatory RNA platforms}

\section{SRNA, RNAi and asRNA}

Various trans-acting regulatory RNAs employ different mechanisms (Table 1). In terms of mode-of-action and target sequences, sRNA, RNAi and asRNA modulate gene expression post-transcriptionally by targeting mRNA molecules, whereas CRISPR exerts transcriptional modulation via interactions with DNA.

In Escherichia coli, Hfq-associated synthetic sRNAs have been implemented for metabolic engineering (Figure 1) [14••]. Hfq is a common RNA-binding protein to facilitate sRNA-mRNA complex formation and mRNA degradation. A modular synthetic sRNA template was designed based on MicC, which is a naturally occurring sRNA in E. coli [14••]. This sRNA template consists of a common scaffold sequence to recruit the Hfq protein, and a custom target-binding sequence that is complimentary to the translation initiation region (TIR) of a given gene to attenuate translational initiation (Figure 1). The versatility of this approach was demonstrated in 14 different E. coli strains, through combinatorial repression of four gene targets, screening of a medium-sized sRNA library, and fine-tuning of knockdown levels [14••]. The ability to regulate gene expression without modification of genome sequences greatly accelerate the prototyping of production hosts.

RNAi is a common gene silencing mechanism in eukaryotes (Figure 2A) [9]. However, the model yeast, Saccharomyces cerevisiae, lacks a native RNAi pathway [13]. The recent reconstitution of RNAi machinery in S. cerevisiae enables the use of this powerful tool for yeast genome engineering [15]. Enzymatically generated genomic DNA fragments were cloned into a pair of convergent constitutive promoters to construct double-stranded RNA libraries for genome-wide silencing (Figure 2B) [16••]. Screening the resultant library led to identification of suppressors of a telomere-defect mutation and genetic 
determinants for improved tolerance towards lignocellulosic hydrolysate inhibitors $[16 \bullet \bullet, 17]$. Furthermore, RNAi-assisted genome evolution (RAGE) enables continuous improvement of a target phenotype, by applying a rationale similar to directed evolution for protein engineering (Figure 2B). During one round of RAGE, several single knockdown modifications were first identified by RNAi screening, and then integrated in the yeast genome to create new parent strains for the next round of engineering (Figure 2B). In this way, multiplex knockdown mutations were accumulated to improve complex traits in S. cerevisiae

$113[16 \bullet \cdot$. The ease of RNAi library construction allows iterative genome-wide screening which is otherwise 114 prohibitively tedious and time-consuming using traditional gene knockout approaches, and therefore lead 115 to discovery of synergistic genetic mutations that are not accessible previously.

116 Compared with sRNA and RNAi, the use of asRNAs have been primarily limited to genome-wide 117 knockdown screening. For example, a paired-termini expression vector was used to express stable asRNAs 118 derived from an E. coli genomic library for a loss-of-function screen [18]. Although also applied in a 119 collection of gram-positive bacteria (as reviewed in [6]) and a yeast pathogen [19], the lack of a clear understanding of gene silencing mechanism by asRNAs hinders the widespread application of asRNA 121 screening.

\section{CRISPR}

123 Whereas most trans-acting RNAs target mRNA molecules, the CRISPR-Cas system provides a RNA124 mediated interference system that cleaves DNA (Figure 3A) [20,21•]. Specifically, the type II Streptococcus pyogenes CRISPR-Cas system offers a simple configuration that contains only a single endonuclease (Cas9) [21•]. Trans-activating CRISPR RNA (tracrRNA) facilitates the processing of

127 CRISPR RNA (crRNA) by Cas9 and the Cas9-crRNA-tracrRNA complex introduces a double stranded 128 break (DSB) at the targeted genome locus (Figure 3A). The tracrRNA and crRNA molecules can also be 129 engineered into a single guide RNA (sgRNA) [21•]. The specificity of target recognition is determined by 130 the guide sequence in the crRNA followed by a short sequence called protospacer adjacent motif (PAM) 
131 [21•]. The DSBs introduced by CRISPR-Cas can substantially improve genome editing efficiency by 132 stimulating the process of non-homologous end joining (NHEJ) or homologous recombination (HR) [22].

133 This versatile framework has already been applied in a wide range of organisms including bacteria [23], 134 yeasts [24], plants [25], animals [26,27], and human cells [22,28].

135 In microbial genome engineering, multiplex genome editing is desirable to accelerate strain prototyping 136 and combinatorial optimization. Traditional approaches rely on sequential incorporation of multiple 137 modifications, which can be very tedious and time-consuming. On the other hand, improved editing 138 efficiency and multiplex nature of the CRISPR-Cas system may help to tackle this challenge. In $S$. cerevisiae, one-step multiple gene disruption was achieved using the Homology-integrated CRISPR-Cas (HI-CRISPR) system (Figure 3B) [29•]. Homologous knockout donors for premature stop codon 141 introduction were inserted before the 5' end of the guide sequences in crRNAs, and such configuration 142 permitted integration of all the necessary CRISPR elements in a single ultrahigh copy plasmid (Figure 3B). 143 Three genes were simultaneously disrupted with an efficiency ranging from $27 \%$ to $100 \%$ after a single 144 transformation in 4-6 days [29•], whereas traditional methods require six rounds of transformation and may 145 take approximately 6 weeks. In another study, a collection of 31 S. cerevisiae strains covering all possible 146 knockout combinations of five genes in an isoprenoid biosynthesis pathway were rapidly constructed via 147 CRISPR [30]. The CRISPR-Cas system was also employed to disrupt multiple alleles of the same gene in 148 polyploid industrial S. cerevisiae strains [31]. Besides gene disruption, multiplex integration was attempted. 149 In S. cerevisiae, six DNA fragments bearing 11 genes for muconic acid biosynthesis were inserted at three 150 genomic loci where DSBs were generated by CRISPR [32]. Although integration efficiency requires 151 further improvement (currently 4.2\%) to reduce the effort in identifying correct constructs, substantial 152 decrease in the time commitment (6-fold in this study) to prototype large biochemical pathways can greatly 153 accelerate pathway engineering [32]. Likewise, multiplex mutagenesis by CRISPR has also been 154 demonstrated in bacteria, including E. coli, Tatumella citrea, Streptococcus pneumonia and Streptomyces $155[23,33,34]$. In particular, the dual cleavage system was recently implemented in chromosomal deletions in 
Streptomyces species, where genomic DNA portions of various sizes (from $20 \mathrm{bp}$ to $30 \mathrm{~kb}$ ) were removed

157 following DSBs generation in two genomic loci [34]. This new capability of rapid, efficient and precise chromosomal editing may greatly facilitate natural product research in actinobacteria.

159 The CRISPR-Cas system has also been expanded for gene regulation without altering the target DNA 160 sequences. To achieve this goal, a deactivated Cas9 (dCas9) protein with abolished endonuclease activity was created (Figure 3A) [35••]. It was found that efficient gene silencing can be achieved when the nontemplate DNA strand was targeted by dCas9/sgRNA complex, which blocked transcriptional initiation or elongation [35••]. This so-called CRISPR interference (CRISPRi) has been successfully demonstrated in E. coli, actinomycetes, yeast and mammalian cells $[35 \bullet \cdot, 36,37]$. Also, fine-tuning of gene repression can be realized via introduction of mismatches between crRNA and target sequences, as well as by variation of target sequences [38]. In addition, when fused with transcription factors, dCas9 can accomplish more modes of gene regulation (Figure 3A). For example, gene activation can be achieved in E. coli and yeast, when dCas9 is fused to the omega subunit of bacterial RNA polymerase and yeast transcriptional activator domains, respectively [36-39]. Likewise, gene silencing efficiency can be improved by fusing dCas9 to transcription repressors or chromatin silencers [36]. Together, the dCas9-based gene regulation platform

171 has demonstrated great versatility in terms of host selection and fine-tuning capability.

\section{Technology advances in RNA-assisted genome engineering}

\section{Computational design}

174 For the computational design of trans-acting regulatory RNAs, one aim is to predict gene modulation 175 efficiency. For example, a quantitative relationship between the repression capability and the binding 176 energy was established for sRNA regulators in E. coli, allowing rational design for tunable gene regulation $177[12 \bullet \cdot]$. Another aim is to design synthetic RNA reagents with optimized targeting efficacy and minimized 178 off-target effect to create genome-wide libraries. For RNAi, in addition to direct construction using 
genomic libraries [14••], it is also possible to computationally design synthetic pools of RNAi sequences, and the readers are directed to a more comprehensive review for available tools [40]. For CRISPR, there are a few web servers that can design target sequences for microbes including E. coli, S. cerevisiae and Aspergillus [41-43]. In particular, E-CRISP ranks targeting sequences of the same gene according to specificity, annotation and efficacy scores, and is able to create genome-scale libraries in a few hours thanks to its fast algorithms (http://www.e-crisp.org/E-CRISP/) [41]. CRISPRdirect offers application program interface (API) to allow generation of many targeting sequences in an automated manner, and therefore enables genome-scale design (http://crispr.dbcls.jp/) [43]. Although genome-wide screening using CRISPR has not been demonstrated in microbial hosts, in the future, these computational tools are indispensable to design target sequence libraries on a genome scale.

\section{Context-dependent regulation}

One limitation of trans-acting regulatory RNAs is that they lack the innate ability to sense cellular signals. Such capability may be incorporated in three ways. First, inducible promoters can be used to control the synthesis of regulatory RNAs and their accessory proteins. For example, two components of CRISPRi, dCas9 and guide RNA, were subjected to the control of an inducible promoter, and this configuration linked gene repression to the presence of the inducer [35••]. In another case, the synthesis of a hairpin RNAi construct was driven by quorum-sensing promoters in S. cerevisiae. Dynamic gene knockdown was achieved where the target genes were only silenced when cell culture reached a high population density [44]. Second, allosteric modulation can be implemented by creating a hybrid RNA molecule that consists of both a cis-ligand-sensing motif and a trans-target-recognition motif. Ligand binding led to structural rearrangement that allowed the recognition motif to form a functional conformation [45•]. Third, signal sensing capability can be programmed into the accessory proteins of regulatory RNAs. In a light-activated CRISPR-Cas9 effector (LACE) system, dCas9 and a transcriptional activator domain were fused with cryptochrome 2 (CRY2) and cryptochrome-interacting basic-helix-loop-helix (CIB1) proteins from Arabidopsis thaliana, respectively. Upon blue light irradiation, the heterodimerization of CRY2 and CIB1 
204 recruited the activator domain to dCas9 that binds to a target locus, and therefore achieved spatiotemporal 205 gene activation [46,47]. Furthermore, the development of split Cas9 also permits regulated activation of 206 CRISPR dependent on the inducible assembly of a functional Cas9 [48].

\section{Conclusions}

208 Regulatory RNA-assisted genome engineering has become an emerging platform for large-scale and 209 multiplex modifications of microbial genomes. However, there are certain limitations requiring further 210 development. For example, most RNA regulation mechanisms repress gene expression. Though RNA211 mediated gene overexpression methods are being developed [36-38,49], general principles are still missing 212 to design activating RNAs on a genome scale. Also, off-target effect is a legitimate concern to most RNA 213 platforms $\left[9,10^{\bullet \bullet}, 19 \bullet\right]$, and therefore precautions have to be taken when interpreting genome-wide 214 screening results.

215 In the future, the discovery and adaptation of more RNA regulatory mechanisms will provide a rich source 216 to develop new engineering platforms. In addition, although genome-wide screening using CRISPR in 217 microorganisms has not been reported, the practice of CRISPR-mediated functional screening in human 218 cells clearly shows the potential [50]. Moreover, the application of RNA-mediated genome engineering in 219 less-studied microbial hosts will facilitate the development of new chassis. Together, we foresee an 220 accelerating trend in implementing regulatory RNAs in the understanding and engineering of microbial 221 genomes. 


\section{Acknowledgements}

223 We gratefully acknowledge financial support from Defense Advanced Research Program Agency

224 (HR0011-14-C-0069), Roy J. Carver Charitable Trust (13-4257), and Institute for Genomic Biology at the

225 University of Illinois at Urbana-Champaign for financial support in our development and application of 226 genome-scale engineering tools.

227 


\section{References and recommended reading}

Papers of particular interest, published within the period of review, have been highlighted as:

- of special interest

•• of outstanding interest

1. Keasling JD: Manufacturing molecules through metabolic engineering. Science 2010, 330:1355-1358.

2. Rabinovitch-Deere CA, Oliver JWK, Rodriguez GM, Atsumi S: Synthetic biology and metabolic engineering approaches to produce biofuels. Chem Rev 2013, 113:4611-4632.

3. Lee JW, Na D, Park JM, Lee J, Choi S, Lee SY: Systems metabolic engineering of microorganisms for natural and non-natural chemicals. Nat Chem Biol 2012, 8:536-546.

4. Carr PA, Church GM: Genome engineering. Nat Biotechnol 2009, 27:1151-1162.

5. Esvelt KM, Wang HH: Genome-scale engineering for systems and synthetic biology. Mol Syst Biol 2013, 9:641.

6. Si T, Xiao H, Zhao H: Rapid prototyping of microbial cell factories via genome-scale engineering. Biotechnol Adv 2014, DOI:10.1016/j.biotechadv.2014.11.007

7. Segal DJ, Meckler JF: Genome engineering at the dawn of the golden age. Annu Rev Genomics Hum Genet 2013, 14:135-158.

8. Jeong J, Cho N, Jung D, Bang D: Genome-scale genetic engineering in Escherichia coli. Biotechnol Adv 2013, 31:804-810.

9. Mohr SE, Smith JA, Shamu CE, Neumuller RA, Perrimon N: RNAi screening comes of age: improved techniques and complementary approaches. Nat Rev Mol Cell Biol 2014, 15:591-600.

10••. Qi LS, Arkin AP: A versatile framework for microbial engineering using synthetic non-coding RNAs. Nat Rev Microbiol 2014, 12:341-354.

10 $\bullet$. A comprehensive review on the design and application of non-coding RNA devices in microbial engineering.

11. Chappell J, Takahashi MK, Meyer S, Loughrey D, Watters KE, Lucks J: The centrality of RNA for engineering gene expression. Biotechnol J 2013, 8:1379-1395.

12. Du J, Yuan Y, Si T, Lian J, Zhao H: Customized optimization of metabolic pathways by combinatorial transcriptional engineering. Nucleic Acids Res 2012, 40:e142.

13. Wang HH, Isaacs FJ, Carr PA, Sun ZZ, Xu G, Forest CR, Church GM: Programming cells by multiplex genome engineering and accelerated evolution. Nature 2009, 460:894-898

14••. Na D, Yoo SM, Chung H, Park H, Park JH, Lee SY: Metabolic engineering of Escherichia coli using synthetic small regulatory RNAs. Nat Biotechnol 2013, 31:170-174.

14••. The first example of rationally designed sRNA platform in E. coli for metabolic engineering applications.

15. Drinnenberg IA, Weinberg DE, Xie KT, Mower JP, Wolfe KH, Fink GR, Bartel DP: RNAi in budding yeast. Science 2009, 326:544-550.

16••. Si T, Luo Y, Bao Z, Zhao H: RNAi-Assisted Genome Evolution in Saccharomyces cerevisiae for complex phenotype engineering. ACS Synth Biol 2015, 4:283-291.

16••. This study presents the first report of RNAi screening in S. cerevisiae. In addition, iterative rounds of RNAi screening enabled the use of directed evolution strategy for genome engineering in yeast. 
17. Xiao H, Zhao H: Genome-wide RNAi screen reveals the E3 SUMO-protein ligase gene $\boldsymbol{S I Z 1}$ as a novel determinant of furfural tolerance in Saccharomyces cerevisiae. Biotechnol Biofuels 2014, 7:78.

18. Meng J, Kanzaki G, Meas D, Lam CK, Crummer H, Tain J, Xu HH: A genome-wide inducible phenotypic screen identifies antisense RNA constructs silencing Escherichia coli essential genes. FEMS Microbiol Lett 2012, 329:45-53.

19. De Backer MD, Nelissen B, Logghe M, Viaene J, Loonen I, Vandoninck S, de Hoogt R, Dewaele S, Simons FA, Verhasselt $\mathrm{P}$, et al.: An antisense-based functional genomics approach for identification of genes critical for growth of Candida albicans. Nat Biotechnol 2001, 19:235241.

20. Barrangou R, Fremaux C, Deveau H, Richards M, Boyaval P, Moineau S, Romero DA, Horvath P: CRISPR provides acquired resistance against viruses in prokaryotes. Science 2007, 315:17091712.

21 • Doudna JA, Charpentier E: Genome editing. The new frontier of genome engineering with CRISPR-Cas9. Science 2014, 346:1258096.

21•. An updated, comprehensive review on the CRISPR-Cas9 system.

22. Mali P, Yang L, Esvelt KM, Aach J, Guell M, DiCarlo JE, Norville JE, Church GM: RNA-guided human genome engineering via Cas9. Science 2013, 339:823-826.

23. Jiang W, Bikard D, Cox D, Zhang F, Marraffini LA: RNA-guided editing of bacterial genomes using CRISPR-Cas systems. Nat Biotechnol 2013, 31:233-239.

24. DiCarlo JE, Norville JE, Mali P, Rios X, Aach J, Church GM: Genome engineering in Saccharomyces cerevisiae using CRISPR-Cas systems. Nucleic Acids Res 2013, 41:4336-4343.

25. Shan Q, Wang Y, Li J, Zhang Y, Chen K, Liang Z, Zhang K, Liu J, Xi JJ, Qiu J-L, et al.: Targeted genome modification of crop plants using a CRISPR-Cas system. Nat Biotech 2013, 31:686688.

26. Wang H, Yang H, Shivalila CS, Dawlaty MM, Cheng AW, Zhang F, Jaenisch R: One-step generation of mice carrying mutations in multiple genes by CRISPR/Cas-mediated genome engineering. Cell 2013, 153:910-918.

27. Hwang WY, Fu YF, Reyon D, Maeder ML, Tsai SQ, Sander JD, Peterson RT, Yeh JRJ, Joung JK: Efficient genome editing in zebrafish using a CRISPR-Cas system. Nat Biotechnol 2013, 31:227-229.

28. Cong L, Ran FA, Cox D, Lin SL, Barretto R, Habib N, Hsu PD, Wu XB, Jiang WY, Marraffini LA, et al.: Multiplex genome engineering using CRISPR/Cas systems. Science 2013, 339:819-823.

29•. Bao Z, Xiao H, Liang J, Zhang L, Xiong X, Sun N, Si T, Zhao H: Homology Integrated CRISPRCas (HI-CRISPR) system for one-step multi-gene disruptions in Saccharomyces cerevisiae. ACS Synth Biol 2014, DOI: 10.1021/sb500255k.

29•. This study implemented an intelligent design where homologous donors were inserted before the 5' end of guide sequences in the crRNA array, allowing one-step delivery of all necessary CRISPR elements on a single plasmid into yeast cells for multi-gene knockout.

30. Jakociunas T, Bonde I, Herrgard M, Harrison SJ, Kristensen M, Pedersen LE, Jensen MK, Keasling JD: Multiplex metabolic pathway engineering using CRISPR/Cas9 in Saccharomyces cerevisiae. Metab Eng 2015, 28:213-222.

31. Stovicek V, Borodina I, Forster J: CRISPR-Cas system enables fast and simple genome editing of industrial Saccharomyces cerevisiae strains. Metab Eng Commun 2015, 2:13-22.

32. Horwitz Andrew A, Walter Jessica M, Schubert Max G, Kung Stephanie H, Hawkins K, Platt Darren M, Hernday Aaron D, Mahatdejkul-Meadows T, Szeto W, Chandran Sunil S, et al.: Efficient 
multiplexed integration of synergistic alleles and metabolic pathways in yeasts via CRISPRCas. Cell Systems 2015, DOI: 10.1016/j.cels.2015.02.001.

33. Jiang Y, Chen B, Duan C, Sun B, Yang J, Yang S: Multigene editing in the Escherichia coli genome via the CRISPR-Cas9 system. Appl Environ Microbiol 2015, 81:2506-2514.

34. Cobb RE, Wang Y, Zhao H: High-efficiency multiplex genome editing of Streptomyces species using an engineered CRISPR/Cas system. ACS Synth Biol 2014, DOI: 10.1021/sb500351f.

35••. Qi LS, Larson MH, Gilbert LA, Doudna JA, Weissman JS, Arkin AP, Lim WA: Repurposing CRISPR as an RNA-guided platform for sequence-specific control of gene expression. Cell 2013, 152:1173-1183.

35••. The inception of CRISPR interference (CRISPRi). Reversible gene regulation is enabled using a catalytic inactive Cas9 to interfere with transcriptional processes.

36. Gilbert LA, Larson MH, Morsut L, Liu Z, Brar GA, Torres SE, Stern-Ginossar N, Brandman O, Whitehead EH, Doudna JA, et al.: CRISPR-mediated modular RNA-guided regulation of transcription in eukaryotes. Cell 2013, 154:442-451.

37. Tong Y, Charusanti P, Zhang L, Weber T, Lee SY: CRISPR-Cas9 based engineering of Actinomycetal genomes. ACS Synth Biol 2015. DOI: 10.1021/acssynbio.5b00038.

38. Bikard D, Jiang W, Samai P, Hochschild A, Zhang F, Marraffini LA: Programmable repression and activation of bacterial gene expression using an engineered CRISPR-Cas system. Nucleic Acids Res 2013, 41:7429-7437.

39. Farzadfard F, Perli SD, Lu TK: Tunable and multifunctional eukaryotic transcription factors based on CRISPR/Cas. ACS Synth Biol 2013, 2:604-613.

40. Lagana A, Shasha D, Croce CM: Synthetic RNAs for gene regulation: Design principles and computational tools. Front Bioeng Biotechnol 2014, 2:65.

41. Heigwer F, Kerr G, Boutros M: E-CRISP: fast CRISPR target site identification. Nat Methods 2014, 11:122-123

42. Naito $\mathrm{Y}$, Hino $\mathrm{K}$, Bono $\mathrm{H}$, Ui-Tei $\mathrm{K}$ : CRISPRdirect: software for designing CRISPR/Cas guide RNA with reduced off-target sites. Bioinformatics 2015, 31:1120-1123.

43. Bae S, Park J, Kim JS: Cas-OFFinder: a fast and versatile algorithm that searches for potential off-target sites of Cas9 RNA-guided endonucleases. Bioinformatics 2014, 30:1473-1475.

44. Williams TC, Averesch NJ, Winter G, Plan MR, Vickers CE, Nielsen LK, Kromer JO: Quorumsensing linked RNA interference for dynamic metabolic pathway control in Saccharomyces cerevisiae. Metab Eng 2015, 29:124-134.

45•. Qi L, Lucks JB, Liu CC, Mutalik VK, Arkin AP: Engineering naturally occurring trans-acting noncoding RNAs to sense molecular signals. Nucleic Acids Res 2012, 40:5775-5786.

45•. This work created RNA fusions combining trans-acting and cis-acting modules, which permit the integration of sensing and targeting capabilities of RNA devices

46. Polstein LR, Gersbach CA: A light-inducible CRISPR-Cas9 system for control of endogenous gene activation. Nat Chem Biol 2015, 11:198-200.

47. Nihongaki Y, Yamamoto S, Kawano F, Suzuki H, Sato M: CRISPR-Cas9-based photoactivatable transcription system. Chem Biol 2015, 22:169-174.

48. Wright AV, Sternberg SH, Taylor DW, Staahl BT, Bardales JA, Kornfeld JE, Doudna JA: Rational design of a split-Cas9 enzyme complex. Proc Natl Acad Sci U S A 2015, 112:2984-2989.

49. Chappell J, Takahashi MK, Lucks JB: Creating small transcription activating RNAs. Nat Chem Biol 2015, 11:214-220.

50. Wang T, Wei JJ, Sabatini DM, Lander ES: Genetic screens in human cells using the CRISPR-Cas9 system. Science 2014, 343:80-84. 
372 Table 1. Comparison of different trans-acting RNA platforms in microbial genome engineering

\begin{tabular}{|c|c|c|c|c|c|c|}
\hline SRNA & $\begin{array}{c}\text { Host } \\
\text { range }\end{array}$ & $\begin{array}{c}\text { Target } \\
\text { substrate }\end{array}$ & $\begin{array}{c}\text { Accessory } \\
\text { protein(s) }\end{array}$ & $\begin{array}{c}\text { Mode of } \\
\text { action }\end{array}$ & $\begin{array}{c}\text { Gene } \\
\text { expression } \\
\text { modulation }\end{array}$ & $\begin{array}{c}\text { Library } \\
\text { creation from } \\
\text { genomic } \\
\text { DNA/cDNA }\end{array}$ \\
\hline RNAi & Eukaryote & RNA & RISC & $\begin{array}{c}\text { Post- } \\
\text { transcriptional }\end{array}$ & Knockdown & Yes \\
\hline asRNA & $\begin{array}{c}\text { Prokaryote } \\
\text { and } \\
\text { Eukaryote }\end{array}$ & RNA & Diverse & $\begin{array}{c}\text { Post- } \\
\text { transcriptional } \\
\text { and } \\
\text { overexpression }\end{array}$ & Knockdown & Yes \\
\hline $\begin{array}{c}\text { CRISPR- } \\
\text { Cas }\end{array}$ & $\begin{array}{c}\text { Prokaryote } \\
\text { and } \\
\text { Eukaryote }\end{array}$ & DNA & Cas9 & Transcriptional & $\begin{array}{c}\text { Knockdown, } \\
\text { overexpression } \\
\text { and knockout }\end{array}$ & No \\
\hline
\end{tabular}




\section{$375 \quad$ Figure legends}

376 Figure 1. Mechanisms of translation repression by Hfq-associated synthetic sRNA. The sRNA template

377 consists of a stem-loop scaffold for Hfq protein recruitment and a target-binding sequence to interfere the

378 binding of ribosome to translation initiation regions (TIRs) of the mRNA molecules.

379 Figure 2. Mechanism and application of RNAi in S. cerevisiae. (A) RNA-induced silencing complex 380 (RISC) seeks and degrades mRNA targets under the guide of small interfering RNAs (siRNAs), and 381 therefore leads to gene silencing. (B) In RNAi-assisted genome engineering (RAGE), yeast genomic DNA 382 fragments are inserted between a pair of convergent promoters to synthesize long double-stranded RNAs 383 for RISC activation. Then, following genome-wide RNAi screening, the identified beneficial cassettes 384 conferring improved phenotypes are integrated to create new parent strains for the next round of RAGE. 385 Iterative rounds of RAGE result in accumulation of multiplex knockdown targets for directed genome 386 evolution $[14 \cdot \bullet]$

387 Figure 3. Mechanism and application of CRISPR in microbial genome engineering. (A) In the CRISPR388 Cas system, the Cas9 protein recognizes and cleaves target DNAs using crRNAs or sgRNAs as guide 389 sequences. Endonuclease-inactive Cas9 (dCas9) can be fused with transcriptional repression and activation 390 domains (RDs and ADs) to regulate gene expression. (B) In the Homology-integrated CRISPR-Cas (HI391 CRISPR) system, triple knockouts are obtained via introduction of an array of crRNAs creating three DSBs 392 simultaneously in the yeast genomes, followed by homologous recombination repair with the donors 393 embedded in the crRNA array [27•]. 
Figure 1

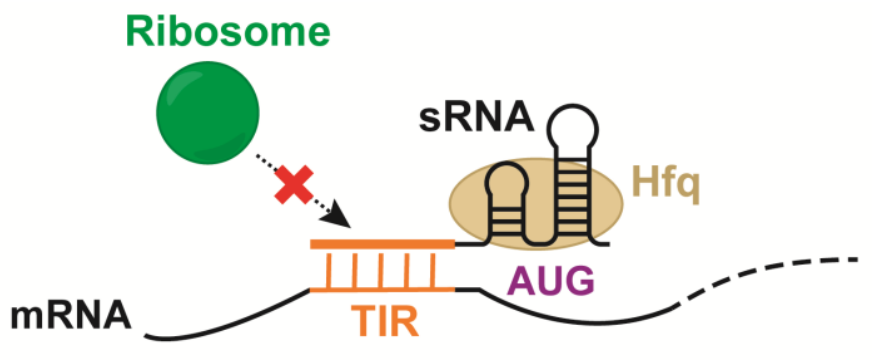

396

397 
398 Figure 2

A

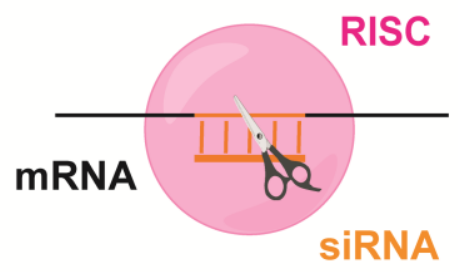

B

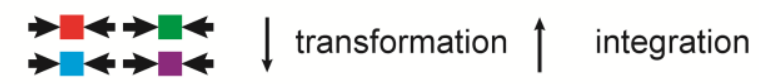

\|\| 10

\|\|$-\|\|$ screen

399

400 
401 Figure 3.

A trRNA+crRNA (or sgRNA)

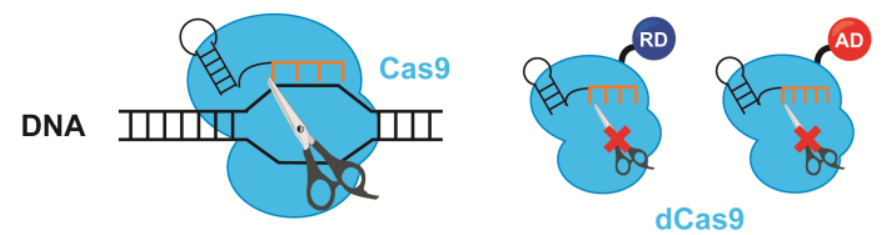

B

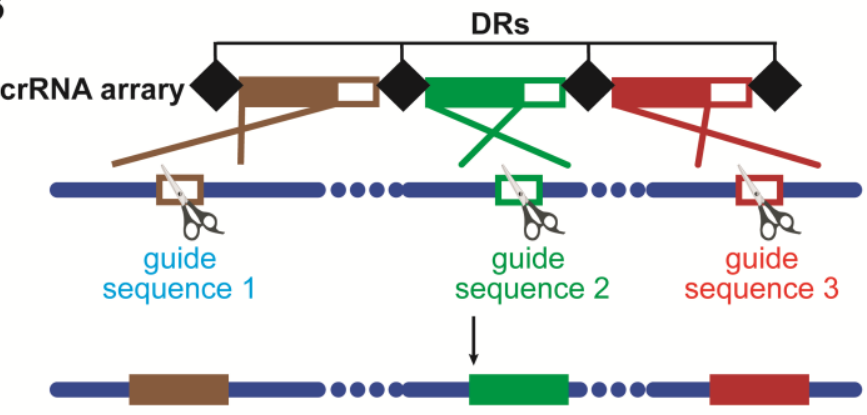

$\begin{array}{lll}\text { HR donor } 1 & \text { HR donor } 2 & \text { HR donor } 3\end{array}$

402 